\title{
COMUNICAÇÃO
}

\section{TEORES DO ÓLEO ESSENCIAL DE CIDRÃO [Aloysia triphylla (L'Hérit) Britton (Verbenaceae)] EM DIFERENTES HORÁRIOS DE COLHEITA E PROCESSAMENTOS PÓS-COLHEITA}

\author{
Lemon verbena's [Aloysia triphylla (L’Hérit) Britton (Verbenaceae)] essential \\ oil content in different harvest periods and post-harvesting process \\ Renata da Silva Brant ${ }^{1}$, José Eduardo Brasil Pereira Pinto ${ }^{2}$, \\ Suzan Kelly Vilela Bertolucci ${ }^{3}$, Andréa da Silva ${ }^{4}$, Carlos Juliano Brant Albuquerque ${ }^{5}$
}

\begin{abstract}
RESUMO
Este estudo foi realizado com o objetivo de avaliar o teor do óleo essencial de cidrão [Aloysia triphylla (L’Hérit) Britton] em diferentes horários de colheita e processamentos pós-colheita. O delineamento utilizado foi inteiramente casualizado. As colheitas foram realizadas em três horários distintos $(8 \mathrm{~h}, 12 \mathrm{~h}$ e $16 \mathrm{~h})$, com três repetições. Os processamentos pós-colheita foram cinco: T1folhas frescas fragmentadas em 1cm; T2- folhas frescas processadas em liquidificador; T3- folhas frescas inteiras; T4- folhas secas inteiras; T5- folhas secas pulverizadas em moinho, com três repetições. O óleo essencial de cada tratamento foi extraído pela técnica de hidrodestilação, utilizando o aparelho modificado de Clevenger, por uma hora e trinta minutos. Os horários de colheita quando se detectou maior teor de óleo foram $8 \mathrm{~h} \mathrm{e} 16 \mathrm{~h}$. Os maiores teores de óleo essencial foram encontrados em folhas frescas fragmentadas em $1 \mathrm{~cm}$, folhas frescas processadas em liquidificador, folhas frescas inteiras e folhas secas inteiras.
\end{abstract}

Termos para indexação: Aloysia triphylla, planta medicinal, metabólito secundário.

\section{ABSTRACT}

This study was carried out in order to evaluate the essential oil content of lemon verbena at different harvest times and postharvest processings. The experimental design used was completely randomized. The harvested material was collected at three different hours $(8: 00,12: 00$ and 16:00 pm), and it was taken three times. In post-harvest processings, three replications and five treatments were us do: $1 \mathrm{~cm}$ fresh leaf fragments, blended fresh leaves, whole fresh leaves, whole dry leaves and dry leaves ground in mill. The essential oil was determined in Clevenger's modified apparatus for 1 hour and 30 minutes. The periods of the day that detected high essential oil content were at 8:00 am and 16:00 pm. The high essential oil level was in $1 \mathrm{~cm}$ fresh leaf fragments, blender processed fresh leaves, whole fresh leaves and whole dry leaves.

Index terms: Aloysia triphylla, medicinal plant, secondary metabolite.

(Recebido em 30 de novembro de 2006 e aprovado em 11 de junho de 2007)

Aloysia triphylla possui os nomes populares: cidrão, erva-cidreira, cidró, cidró-pessegueiro, erva-luísa e cidrozinho. É um arbusto grande, de dois a três metros de altura, muito ramificado e ereto (LORENZI \& MATOS, 2002).

Suas folhas são empregadas internamente contra resfriados febris, como tônico, antiespasmódico, carminativo, eupéptico e calmante. Em aromaterapia são empregadas contra problemas nervosos e para acnes. Podem ser usadas para tratamento de melancolia, afecções do coração e histeria, sendo também emenagoga (LORENZI \& MATOS, 2002).

Identificar as produções de óleo essencial ao longo do dia, selecionando o melhor momento para a colheita é de suma importância para o produtor de aromas (MARTINS et al., 1994), pelo fato de ocorrerem, durante o dia, oscilações

1Pós-doutoranda em Agronomia - Instituto de Ciências Agrárias/ICIAG - Universidade Federal de Uberlândia/UFU - Cx. P. 597 - Avenida Pará, sala 14 Campus Umuarama - 38400-902 - Uberlândia, MG - renataplantasmedicinais@yahoo.com.br

${ }^{2} \mathrm{PhD}$ em Agronomia/Fitotecnia, Professor Titular - Departamento de Agricultura/DAG - Universidade Federal de Lavras/UFLA - Cx. P. 3037 - 37200000 - Lavras - jeduardo@ufla.br

${ }^{3}$ Doutora em Farmacêutica/Química, Professora Adjunta - Departamento de Agricultura/DAG - Universidade Federal de Lavras/UFLA - Cx. P. 3037 37200-000 - Lavras,MG - suzan@ufla.br

${ }^{4}$ Gestora em Agronegócio e Administradora de Empresas - Universidade de Uberaba/UNIUBE - Rua Coronel Severiano, 251 - Tabajaras - $38400-322$ Uberlândia, MG - andrea adm83@yahoo.com.br

${ }^{5}$ Doutor em Agronomia/Fitotecnia, Pesquisador - Empresa Agropecuária de Minas Gerais/EPAMIG-URTMAP- BR 050 - Km 63 - Cx. P. 2248 - $38402-019$ Uberlândia, MG - carlosjuliano@epamig.br 
nos componentes climáticos (principalmente temperatura e luminosidade).

Lippia alba (Mill.) N.E. Brown. (erva-cidreirabrasileira, quimiotipo carvona-limoneno), espécie da mesma família de Aloysia triphylla, apresentou teores de óleo essencial em folhas frescas, colhendo-as às 14 e 16 horas. A colheita nos horários de 08, 10, 12 e 18 horas resultou em teores significativamente menores (EHLERT et al., 2003).

Na colheita de grande volume de fitomassa e não havendo estrutura física para extração imediata de todo o material, é inevitável e imprescindível que ocorra sua secagem, pois esta promove a estabilização do metabolismo da planta, imobilizando a ação enzimática degradadora dos princípios ativos existentes e evita ataque de microrganismos (CORRÊA JÚNIOR et al., 1994).

Para se obter maior eficiência na extração do óleo essencial pode-se fragmentar os órgãos vegetais de diferentes formas, visando ao maior contato superficial destes com o solvente utilizado no processo extrativo e, assim, maior eficiência de extração, aumentando os lucros do produtor. Além disso, caracterizam-se por serem técnicas rápidas, de fácil execução e baixo custo. Costa et al. (2005) concluíram que, pulverizando as folhas de Cymbopogon citratus (D.C.) Stapf. em moinho, haverá maior rendimento de óleo essencial e citral. Após secas em estufa, na fragmentação a pó, o conteúdo de citral foi, em média 16,2\%, maior que na fragmentação de $20 \mathrm{~cm}$ e 4,8\% maior em relação ao corte de $1 \mathrm{~cm}$.

Pela carência de informações relacionadas ao assunto, reportando-nos à espécie em questão e sua relevância, objetivou-se neste trabalho quantificar o teor de óleo essencial das folhas de Aloysia triphylla em diferentes horários de colheita e processamentos póscolheita.

O experimento foi conduzido em campo experimental do Setor de Olericultura da Universidade Federal de Lavras (UFLA), e no Laboratório de Cultura de Tecidos e Plantas Medicinais, Aromáticas e Condimentares do Departamento de Agricultura da UFLA, em Lavras-MG. O município de Lavras está situado na região Sul do estado de Minas Gerais, a 918,87 metros de altitude, latitude sul de $21^{\circ} 14^{\prime} \mathrm{S}$ e longitude oeste de $45^{\circ} 00^{\prime}$ GRW.

A identificação taxonômica da espécie em estudo foi efetuada e as exsicatas foram incorporadas no Herbário ESAL do Departamento de Biologia da UFLA e correspondem ao $\mathrm{n}^{\circ} 19677$.

Microestacas apicais de $A$. triphylla foram retiradas de uma planta matriz e colocadas para enraizar em bandejas de poliestireno de 72 células, contendo o substrato comercial.
Após 62 dias, 60 mudas com $10 \mathrm{~cm}$ de altura foram transplantadas para o campo em solo classificado como Latossolo Vermelho Distroférrico. As mudas foram plantadas em delineamento inteiramente ao acaso, em covas de $30 \times 30 \times 30 \mathrm{~cm}$ que continham $3 \mathrm{~L}$ de composto orgânico por cova, espaçadas em 1,0 x 1,0 m e, após 76 dias foi repetido este procedimento de adubação.

Nos dias 1, 2 e 3 de novembro de 2004 (357, 358 e 359 dias após o transplantio), as folhas de A. triphylla foram coletadas de 60 plantas (em amostragem composta) em diferentes horários do dia $(8 \mathrm{~h}, 12 \mathrm{~h}$ e $16 \mathrm{~h})$ para determinação do teor dos óleos essenciais.

Para a extração dos óleos essenciais dos tratamentos, selecionaram-se apenas as folhas basais, apicais e medianas sadias. Foram utilizadas três repetições, sendo cada dia correspondente a uma repetição.

A massa padronizada foi de 40 gramas de folhas frescas fragmentadas em frações de aproximadamente $1 \mathrm{~cm}$ e submetidas à técnica de hidrodestilação pelo aparelho modificado de Clevenger. O tempo de extração foi de uma 1h30. Após a obtenção do óleo essencial puro, a massa e o teor de óleo foram calculados pela seguinte fórmula: $\mathrm{T} \%=$ Massa do óleo (g) x $100 / 40 \mathrm{~g}$.

Ao final de 192 dias após o transplantio (20 de maio de 2003) foram coletadas às $8 \mathrm{~h}$ da manhã, folhas de 60 plantas de A. triphylla. Posteriormente, as folhas foram levadas para o laboratório, onde realizou-se uma seleção, descartando-se as folhas injuriadas. Para cada tratamento foram utilizadas três repetições e os tratamentos foram: $\mathrm{T} 1$ - folhas frescas fragmentadas em $1 \mathrm{~cm}$; T2- folhas frescas processadas em liquidificador; T3- folhas frescas inteiras; T4- folhas secas inteiras e T5- folhas secas pulverizadas em moinho. A secagem das folhas foi realizada à sombra, por 20 dias.

Para a extração dos óleos essenciais, foram utilizadas $40 \mathrm{~g}$ de folhas frescas (por repetição) e, para folhas secas, foi feita uma correlação entre a biomassa fresca e seca, em que $40 \mathrm{~g}$ de folhas frescas corresponderam, em média, a $15,25 \mathrm{~g}$ de folhas secas.

Para a extração do óleo foi utilizado o método de hidrodestilação em aparelho modificado de Clevenger. O material vegetal foi colocado em balão volumétrico de 1.000 $\mathrm{mL}$ e acrescentado um volume de $700 \mathrm{~mL}$ de água destilada.

Após a detecção de início de destilação, começouse a cronometrar. Finalizado o período de extração (1h 30 por repetição), o óleo essencial foi separado por meio da partição líquido-líquido em funil de separação, com 3 porções de $25 \mathrm{~mL}$ de diclorometano (cada porção descansou por 20 minutos, totalizando 60 minutos por repetição). As frações orgânicas de cada repetição foram reunidas e secas 
com porção em excesso de sulfato de magnésio anidro. $\mathrm{O}$ sal foi removido por filtração simples e o solvente evaporado à temperatura ambiente em capela de exaustão de gases até alcançar peso constante, obtendo-se o óleo essencial purificado.

A análise estatística dos dados foi realizada pelo programa SANEST (ZONTA \& MACHADO, 1984). As médias dos tratamentos foram submetidas à análise de variância pelo teste F e Tukey a 5\% de probabilidade.

$\mathrm{O}$ teste de Tukey detectou diferença estatística significativa, a $5 \%$ de probabilidade, entre os horários de colheita ( $8 \mathrm{~h}, 12 \mathrm{~h}$ e $16 \mathrm{~h}$ horas) das folhas de Aloysia triphylla.

Em horários que apresentam temperaturas mais baixas durante o dia, 8 e 16 horas, observou-se que os teores de óleo essencial foram superiores e estatisticamente iguais, quando comparados ao material coletado ao meiodia, em que o teor médio de óleo essencial reduziu. Este menor valor pode ser creditado á temperaturas mais elevadas às 12 horas (Figura 1).

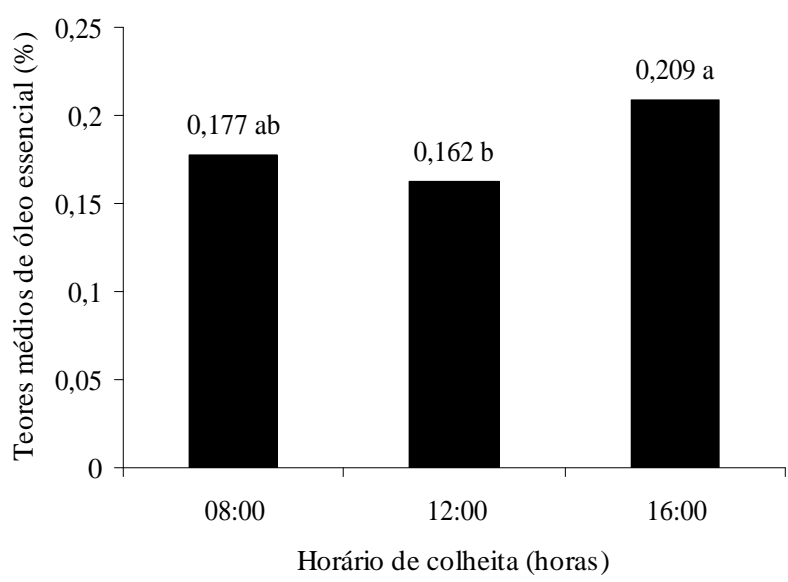

Figura 1 - Teores médios de óleo essencial de folhas frescas de Aloysia triphylla em diferentes horários de colheita. Médias seguidas pela mesma letra não diferem entre si, pelo teste de Tukey, a 5\% de probabilidade.

Estes resultados estão de acordo com os de Simões et al. (1999), que afirmam que, preferencialmente, deve-se coletar plantas ricas em óleos essenciais bem cedo pela manhã ou à noite, pois o período de exposição ao sol pode provocar uma perda quantitativa importante do óleo existente no vegetal.

Em espécies da mesma família de Aloysia triphylla, como Lippia alba (quimiotipo citral-limoneno), a colheita às $15 \mathrm{~h}$, tanto na estação seca quanto na chuvosa, resultou em maior teor de óleo e de citral (constituinte majoritário)
(NAGAO et al., 2004). Em Cordia curassavica (Jacq.) Roen. \& Schult., não houve diferença significativa no teor de óleo de folhas coletadas às 9, 12, 15 e 18 horas (CORRÊA et al., 2002).

Diferença significativa entre os processos de fracionamento adotados foi observada (Figura 2). Quando as folhas foram dissecadas e pulverizadas em moinho em pequenos fragmentos, houve perda significativa de óleo essencial $(0,055 \%)$. Os tricomas capitados e peltados são responsáveis pela síntese do óleo de A. triphylla (SUAREZ et al., 2003) e localizam-se na superfície foliar. Diante disso, pode-se inferir que quando fragmentam-se muito as folhas de A. triphylla, o óleo essencial, localizado na superfície foliar, volatiliza-se no momento do fracionamento.

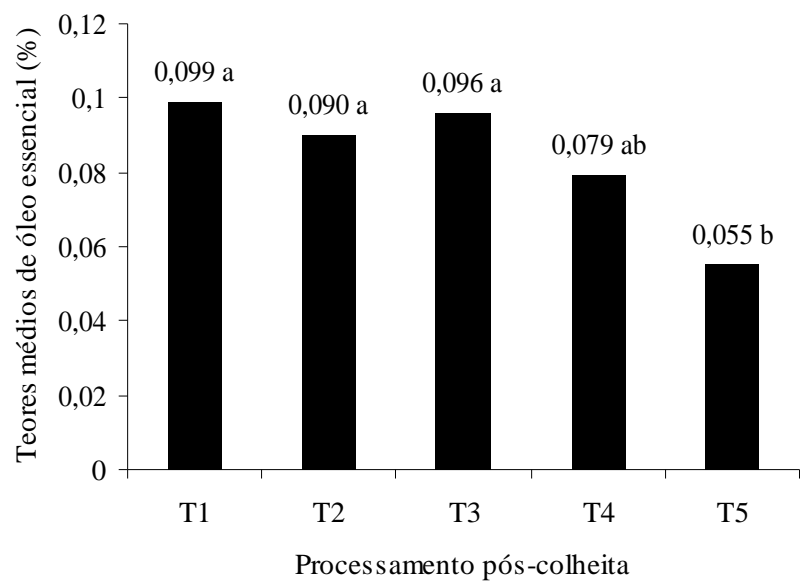

Figura 2 - Teores médios de óleo essencial de Aloysia triphylla (T1- folhas frescas fragmentadas em 1cm; T2folhas frescas processadas em liquidificador; T3- folhas frescas inteiras; T4- folhas secas inteiras; T5- folhas secas pulverizadas em moinho). Médias seguidas pela mesma letra não diferem entre si, pelo teste de Tukey, a 5\% de probabilidade.

Santos (2000), trabalhando com pimenta negra (Piper nigrum L.), pimenta câpsico (Capsicum frutescens L.), cardamomo (Elettaria cardamomum (L.) Maton) e cravo (Caryophilus aromaticus L.), relata que, após a moagem convencional, houve perda no teor de óleo essencial de $34,3 \%, 15 \%, 40 \%$ e $34 \%$, respectivamente. Ademais, acrescenta que o processo de moagem envolve geração de calor, o que pode resultar na perda de componentes voláteis.

Não houve detecção de diferença estatística entre folhas frescas fragmentadas em $1 \mathrm{~cm}$, frescas inteiras, frescas processadas em liquidificador e secas inteiras. 
Salienta-se que os resultados encontrados apontam para cuidados no trato com as plantas de A. triphylla durante o processo de colheita e pós-colheita, pois, pelas estruturas de síntese e estocagem dos óleos estarem na superfície foliar, o amassamento ou estilhaçamento das folhas ocasionam a volatilização do óleo. Deve-se empregar manuseio delicado, evitar acondicioná-las em altas camadas e em recipientes que promovam temperaturas mais elevadas e sem arejamento.

Os horários do dia e processamentos pós-colheita indicados para a espécie Aloysia triphylla para obter as maiores concentrações médias de óleo essencial foram às 8 e 16 horas e em folhas frescas fragmentadas em $1 \mathrm{~cm}$, frescas processadas em liquidificador, frescas inteiras $\mathrm{e}$ secas inteiras.

\section{AGRADECIMENTOS}

Ao CNPq, pela concessão de bolsa.

\section{REFERÊNCIAS BIBLIOGRÁFICAS}

CORREA JÚNIOR, C.; MING, L. C.; SHEFFER, M. C. Cultivo de plantas medicinais, condimentares e aromáticas. 2. ed. Jaboticabal: Fundação de Estudos e Pesquisa em Agronomia, Medicina Veterinária e Zootecnia, 1994.

CORRÊA, R. M.; BERTOLUCCI, S. K. V.; CARDOSO, M. G.; PINTO, J. E. B. P.; REIS, E. S.; CHAGAS, J. H.

Rendimento de caracterização no infravermelho de óleos essenciais de Cordia verbenaceae em função dos horários de coleta. Horticultura Brasileira, Brasília, v. 20, n. 2, jul. 2002. Suplemento 2.

COSTA, L. C. B.; CORRÊA, R. M.; CARDOSO, J. C. W.; PINTO, J. E. B. P.; BERTOLUCCI, S. K. V.; FERRI, P. H. Secagem e fragmentação da matéria seca no rendimento e composição do óleo essencial de capim-limão.

Horticultura Brasileira, Brasília, v. 23, n. 4, p. 956-959, out./dez. 2005.
EHLERT, P. A. D.; MING, L. C.; MARQUES, M. O. M.; FERNANDES, D. M.; FERRI, A. F.; ROCHA, W. A. T.; MEIRELES, M. Â. A. Efeito do horário de colheita sobre a carvona e o limoneno do óleo essencial de ervacidreira brasileira. Horticultura Brasileira, Brasília, v. 21, n. 2, jul. 2003. Suplemento. CD-ROM.

LORENZI, H.; MATOS, F. S. A. Plantas medicinais do

Brasil: nativas e exóticas. Nova Odessa: Plantarum, 2002.

MARTINS, E. R.; CASTRO, D. M.; CASTELLANI, D. C.; DIAS, J. E. Plantas medicinais. Viçosa: UFV, 1994. 220 p.

NAGAO, E. O.; MARCO, C. A.; INNECCO, R.; MATTOS, S. H.; MEDEIROS FILHO, S.; MARCO, C. A. Efeito do horário de corte sobre o rendimento e constituintes majoritários em óleo essencial de Lippia alba (MILL) N.E.BR, quimiotipo citral-limoneno. Revista Ciência Agrônomica, Fortaleza, v. 35, n. 2, p. 355-360, 2004.

SANTOS, E. V. M. Extração de matérias primas vegetais. In: SHARAPIN, N. Fundamentos de tecnologia de produtos fitoterápicos. Santafé de Bogotá: Colômbia, 2000. p. 34.

SIMÕES, C. M. O.; SCHENKEL, E. P.; GOSMANN, G. Farmacognosia da planta ao medicamento. Porto Alegre: UFRGS/UFSC, 1999. $821 \mathrm{p}$.

SUAREZ, S.; GIL, A.; LORENZO, E. Aloysia citriodora: morphology and density of gladular trichomes, and its relationships with essential oil content. In: SIMPÓSIO BRASILEIRO DE ÓLEOS ESSENCIAIS, 2., 2003, Campinas. Anais... Campinas: Instituto Agronômico, 2003. p. 78.

ZONTA, E. P.; MACHADO, A. A. Sistema de análise estatística para microcomputadores - SANEST. Pelotas: UFPel, 1984. 109 p. 